\title{
Lipids in surgical aerosol as diagnosis biomarkers for discrimination of lung cancer
}

This article was published in the following Dove Press journal:

Cancer Management and Research

\author{
Jianyong Zhang ${ }^{1,2, *}$ \\ Qiaoling Zheng ${ }^{1,3, *}$ \\ Wenxiong Zhang' \\ Nanpeng Wang ${ }^{2}$ \\ Jianjun $\mathrm{Xu}{ }^{\prime}$ \\ Xiaoshu Cheng' \\ Yiping Wei'
}

'Department of Cardiothoracic Surgery, The Second Affiliated Hospital of Nanchang University, Nanchang, Jiangxi 330006, People's Republic of China; ${ }^{2}$ Department of General surgery, The Affiliated Hospital of Guizhou Medical University, Guiyang, Guizhou 550000, People's Republic of China; ${ }^{3}$ Jiangxi Health Vocational College, Nanchang, Jiangxi 330000, People's Republic of China

*These authors contributed equally to this work
Correspondence: Yiping Wei

Department of Cardiothoracic Surgery,

The Second Affiliated Hospital of

Nanchang University, No.I Minde Road, Nanchang, Jiangxi Province 330006, P. R. China

Email weiyip2000@hotmail.com

Nanpeng Wang

Department of General Surgery, The

Affiliated Hospital of Guizhou Medical

University, 28 Guiyi Road, Guiyang,

Guizhou 550000, People's Republic of

China

EmailwwwI3568@I63.com
Introduction: Lung cancer is one of the most common malignancies worldwide, and the main effective treatment is surgical operation to cure this disease. This study assessed the feasibility of surgical aerosol for identification of lung cancer and adjacent normal tissue in surgery.

Methods: In vitro experiments, the surgical aerosol was released when the tissue sample was being cut using a standard electrosurgery handpiece. Surgical smoke was dissolved in methanol by negative-pressure suction and then get to the neutral sprayer for analysis. Multivariate analysis was performed using partial least squares (PLS) analysis in MatLab 2011.

Results: A total of 208 surgical aerosol database entries were obtained from 26 patients. In the cancerous aerosol, relative abundance $(760.61,782.39$, and $789.68 \mathrm{~m} / \mathrm{z}$ ) was increased, while relative abundances of $(756.41 \mathrm{~m} / \mathrm{z})$ was decreased compared with normal-tissue aerosol. After PLS analysis, mass-spectrometry (MS) data for the cancer aerosol showed clear differentiation from normal. Four significant peaks were identified by collision-induced dissociation experiments. The cancerous aerosol showed overexpression of phosphatidylserine (34:2), phosphatidylcholine (36:4), and triacylglycerol (46:2), while phosphatidylcholine (34:3) was decreased. Coupling PLS and extractiveelectrospray-ionization MS analysis of the surgical aerosol data of lung cancer were clearly distinguished from normal.

Conclusion: The surgical aerosol might contain biomarkers for identification of lung cancer and normal tissue.

Keywords: lung cancer, biomarker, surgical aerosol, mass spectrometry

\section{Introduction}

Lung cancer is one of the most common malignancies worldwide, and the main effective treatment is surgical operation to cure this disease. ${ }^{1,2}$ Precision medicine aims to improve patient outcomes. In surgery, incomplete excision leads to local recurrence, while excessive resection can lead to complications such as nearby important-organ injury, dyspnea after surgery, and anoxia postoperation. ${ }^{3}$ Therefore, it is important to identify precise tumor margins during surgery to be confident in resection and accurate prognosis, as well as to minimize loss of healthy tissue. ${ }^{4}$ Surgeons determining tumor margins preoperatively must rely on medical imaging, such as chest radiography, computed tomography, magnetic resonance imaging, and positron-emission tomography, ${ }^{5}$ which are not sufficiently sensitive and accurate and depend on subjective judgment. Intraoperative frozen-section histology was the golden standard to confirm tumor margins and isable to provide results within 30 minutes. However, it is associated with many drawbacks: its timeconsuming (30 minutes) nature considerably lengthens the exposure of the patient to the general-anesthetic and operative risk, and histopathological analysis is also 
subjective (the reliability and precision of cancer diagnosis largely depend on the skills and experience of each doctor). ${ }^{6}$ Intraoperative computed tomography can be used to assess margin status and can be performed within the operating theater, providing direct feedback to the surgeon without the need for specially trained personnel. ${ }^{7}$ However, compared to pathological techniques, it has inferior accuracy and radiation exposure. ${ }^{8}$ Due to the limitations of contemporary intraoperative margin-assessment techniques, a plethora of innovative devices are under development aiming to provide a tool that limits workflow disruption, provides rapid results, optimizes margin control, and reduces local recurrence rates. ${ }^{9}$ Therefore, there is an urgent need for precision benign-tumor (such as granuloma or hamartoma) cancer phenotyping for identification during surgical, therapeutic, and some diagnostic interventions.

In all fields of tumor surgery, surgical aerosols containing cells and molecular information might be an ideal method to diagnose pathological margins. In modern surgery, the electrosurgical process of cutting tissue causes heat to dissipate inside the tissue, resulting in cellular explosion and release of cellular content to the gas phase. The surgical aerosol is wasted, due to the lack of detection methods. Mass spectrometry (MS) is one of the most widely used techniques for the analysis of biological samples and plays an important role in analyzing biological samples across multiple disciplines. ${ }^{10}$ With its many advantagesincluding speed of analysis, high sensitivity, low limit of detection (LOD), and no pretreatment MS is considered a powerful method for analyzing complex mixtures. ${ }^{11,12}$ Recently, a new method of MS analysis of surgical aerosol was introduced: advanced rapid evaporative ionization MS, named "intelligent knife", which is a novel technique to analyze aerosol produced during electrosurgical treatment to provide tissue-specific diagnostics based on lipid profiles. ${ }^{6,13}$ Surgical aerosol analysis by MS may be an alternative to standard frozen-section histology, as MS can provide rich molecular information from the aerosol, which can be correlated with pathology.

In this study, we used extractive electrospray ionization (EESI) $\mathrm{MS}^{14}$ to analyze the aerosol produced during surgery. The aim was to assess its feasibility for the identification of cancer and adjacent normal tissue. With this method, it is possible to analyze surgical aerosol directly to discriminate cancerous tissue from adjacent normal tissue during an operation.

\section{Methods}

\section{Materials}

We used MS (LTQ-XL; Thermo Fisher Scientific, Waltham, MA, USA) and a surgical electric knife (Kaisite; Xi'an, People's Republic of China). Methanol (analytical reagent grade) was obtained from Tedia (Fairfield, OH, USA). The deionized water was homemade.

\section{Patients and sample collection}

From March to September 2017, 26 patients undergoing elective surgical treatment for lung cancer were prospectively recruited at the Second Affiliated Hospital of Nanchang University (Nanchang, People's Republic of China). Written informed consent was obtained from all patients. This study was approved by the Medical Ethics Committee of the hospital's institutional review board. All clinical investigations were conducted according to the principles expressed in the Declaration of Helsinki. Inclusion criteria were adult patients with suspected primary lung cancer undergoing elective surgical resection by either open or thoracoscopy surgery. The patients had no other lung diseases, accompanying malignancies, or history of preoperative chemotherapy or radiotherapy. Patients unable to provide consent or those undergoing emergency surgery were excluded. After resection, surgical specimens were immediately transported fresh to the histopathology department by a member of the research team. Specimen were opened by a trained histopathologist, and fresh biopsies cut from the tumor and normal adjacent mucosa at $5 \mathrm{~cm}$ from the lesion. Then, tissue samples were collected in liquid nitrogen within 5 minutes by the histopathologist and stored at $-80^{\circ} \mathrm{C}$ until in vitro experiments.

\section{Extractive electrospray-ionization mass spectrometry}

EESI uses a solution sprayer and a neutral sprayer. ${ }^{14}$ The electrospray was produced by a solution sprayer when solution (methanol-water mixture, v:v 70:30) at a flow rate of 5 $\mu \mathrm{L} / \mathrm{min}$ using a conventional pumping system (ionizing voltage $+3.0 \mathrm{kV}$ ) was applied to the syringe needle and the nebulizer nitrogen gas flow for the solution sprayer was supplied at a pressure of 1.2 MPa. The surgical aerosol was dissolved in methanol by negative pressure suction and then get to the neutral sprayer for analysis. Analytes of the surgical smoke were ionized depending on liquid-liquid extraction between the colliding microdroplets, and then the 
ionized analytes were subjected to MS. The mass-scan range was set to $700-900 \mathrm{~m} / \mathrm{z}$, capillary temperature to $150^{\circ} \mathrm{C}$, capillary voltage to $10 \mathrm{~V}$, and tube-lens voltage to $100 \mathrm{~V}$.

\section{Ex vivo analysis}

Fresh tissue samples were analyzed ex vivo with an electrosurgery handpiece using monopolar diathermy in cutting mode with aspiration of the electrosurgical aerosol through the neutral sprayer to the LTQ-XL. This process took 5 minutes from capture of MS data to analysis. The same tissue samples underwent histological examination by two trained histopathologists for validation purposes.

\section{Data analysis}

Mass spectra were collected in single-stage MS, positiveion mode, and the mass range was $700-900 \mathrm{~m} / \mathrm{z}$. Four mass spectra were obtained per sample. Raw spectra data obtained from surgical aerosol were exported to Microsoft Excel and arranged according to their $\mathrm{m} / \mathrm{z}$ values with unit resolution (independent variable) and using the relative abundance of full-scan mass fingerprints (dependent variables). All MS data were treated as matrix $\mathrm{X}$, and rows and columns corresponded to sample cases and $\mathrm{m} / \mathrm{z}$-value variables, respectively. The relative abundance of MS data were normalized to reduce the impact of variation on overall signal intensity expressed. All MS data belonging to one patient were left out of the sample set, and a new model was calculated using the remaining data. The withheld data were projected into the new model and classified as one tissue type between the unknown sample point and calculated class centers. This process was repeated four times for each individual sample. The discriminating models were then subjected to dimensionality reduction via partial least squares (PLS) analysis to reduce the number of observed variables importance in projection, which account for the majority of the variance in the data set. To identify statistically significant peaks responsible for PLS classification into each group, the first five were kept for further analysis. Exact $\mathrm{m} / \mathrm{z}$ values were retrieved from the raw data, and these values were used for compound identification via database search.

\section{Results}

A total of 26 patients were recruited for the ex vivo experiments of the study (17 males, nine females). Patient demographics and clinical information of the remaining cases are shown in Table 1.
Table I Demographic and clinical information of enrolled patients.

\begin{tabular}{|l|l|}
\hline Male/female & $17 / 9$ \\
Median age, years (range) & $56(30-65)$ \\
\hline Site of lesion & \\
Upper lobe of right lung & 7 \\
Middle lobe of right lung & 1 \\
Inferior lobe of right lung & 6 \\
Upper lobe of left lung & 8 \\
Inferior lobe of left lung & 4 \\
\hline Histopathology & \\
Squamous-cell carcinoma & 11 \\
Adenocarcinoma & 13 \\
Adenosquamous carcinoma & 2 \\
\hline T stage & \\
TI & 5 \\
T2 & 13 \\
T3 & 8 \\
T4 & 0 \\
\hline N stage & \\
N0 & 19 \\
NI & 6 \\
N2 & 1 \\
\hline N stage & \\
M0 & \\
MI & \\
\hline
\end{tabular}

\section{EESI-MS analysis of surgical aerosol}

Representative mass spectra of the surgical aerosol from cancer and normal tissue can be seen in Figure 1. In the positive-ion mode, the mass range was set at 700-900 Da, main peaks from cancer tissue were 703.49, 725.56, 734.63, 756.58, 760.59, 782.57, 789.59, 791.11, and $810.54 \mathrm{~m} / \mathrm{z}$, while those of adjacent normal tissue were 703.49, 725.59, 734.58, 756.56, 760.57, 782.58, 789.59, and $808.60 \mathrm{~m} / \mathrm{z}$ (Figure 1). In cancerous aerosol, relative abundance values of $760.61,782.39$, and $789.68 \mathrm{~m} / \mathrm{z}$ were increased, while relative abundance $756.41 \mathrm{~m} / \mathrm{z}$ was decreased compared with normal-tissue aerosol (Table 2).

\section{Discrimination of surgical aerosol MS data by PLS}

All MS data expressed asrelative abundance were normalized to reduce the impact of variation in overall signal intensity expressed for PLS analysis. A total of 208 surgical aerosol database entries from 26 patients (each normal and cancerous tissue sample analysed four times) were interpreted in PLS-score graphs. EESI-MS was able 


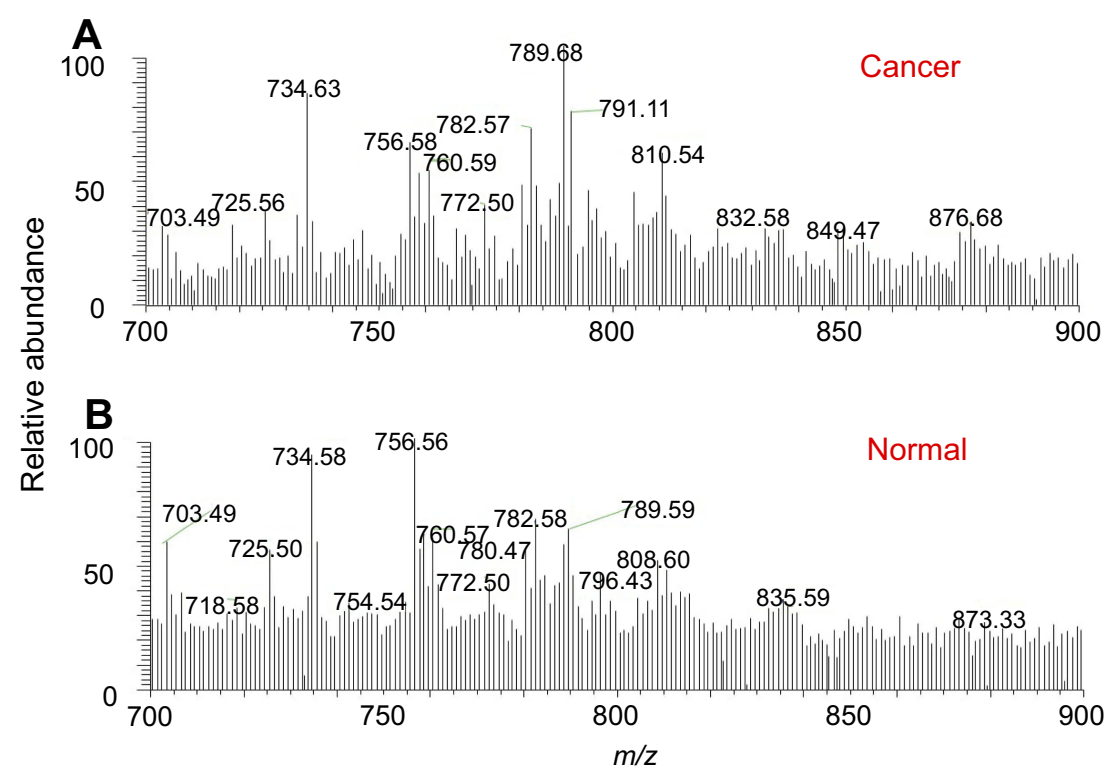

Figure I Example of mass spectra from surgical smoke of cancerous tissue (A) and adjacent normal tissue (B) The 700-900 m/z range has been selected.

Table 2 Summary putative metabolite IDs for surgical aerosol from cancer and normal tissue, their $\mathrm{m} / \mathrm{z}$ and statistical significance (P-value)

\begin{tabular}{|l|l|l|l|l|l|}
\hline $\mathbf{m} / \mathbf{z}$ & Putative molecular structure & P-value & Normal & Cancer & Expression in cancer \\
\hline 756.41 & PC (34:3) & $<0.001$ & $7.44 \pm 2.48$ & $5.92 \pm 1.07$ & Underexpressed \\
760.61 & PS (34:2) & $<0.001$ & $4.86 \pm 0.20$ & $5.46 \pm 0.15$ & Overexpressed \\
782.39 & PC (36:4) & 0.001 & $6.44 \pm 2.35$ & $7.48 \pm 1.56$ & Overexpressed \\
789.68 & TG (46:2) & $<0.001$ & $5.45 \pm 2.02$ & $8.52 \pm 2.04$ & Overexpressed \\
\hline
\end{tabular}

Abbreviations: PC, phosphatidylcholine; PS, phosphatidylserine; TG, triacylglycerol.

reliably to distinguish between normal and cancer tissue (Figure 2). Univariate analysis of target metabolites was performed based on results from the loading data to confirm their statistical contribution to the model and to identify descriptive metabolites responsible for class description (Table 2). This indicated that lipid abundance was protean in cancer tissue. Univariate statistical analysis was used to identify significant peaks that differed between normal and cancerous aerosols. Four significant peaks remained: three peaks were higher and one lower in the cancerous aerosol (Table 2).

\section{Identified discriminatory ions by collision-induced dissociation experiments}

Four differential ions were tentatively identified on the basis of collision-induced dissociation experiments and comparison with standard-product collision-induced dissociation data. The 756, 760, 782, and $789 \mathrm{~m} / \mathrm{z}$ results represented phosphatidylcholine (PC; (34:3), phosphatidylserine (PS; 34:2), PC (36:4), and triacylglycerol (TG; 46:2), respectively (Figure 3 ).

\section{Discussion}

In the field of tumor oncology, identification of tumor margins is crucially important to obtain confidence in curative resection and accurate prognosis, as well as to minimize losses to healthy tissue. ${ }^{4}$ Lung cancer is the most common malignant carcinoma, and surgical treatment is the most efficient way to cure it. ${ }^{15}$ The current goldstandard method to determine tumor margins accurately during surgery is frozen-section histology, but it has some shortcomings. ${ }^{16}$ Firstly, frozen-section histology during surgery takes 30 minutes, which is a long time for the patient and the surgeon. Secondly, the simplified histological processing results in decreased reliability, especially for samples containing high amounts of adipose tissue. Lastly, the histological diagnosis is established based on the visual perception of morphological tissue features, and 

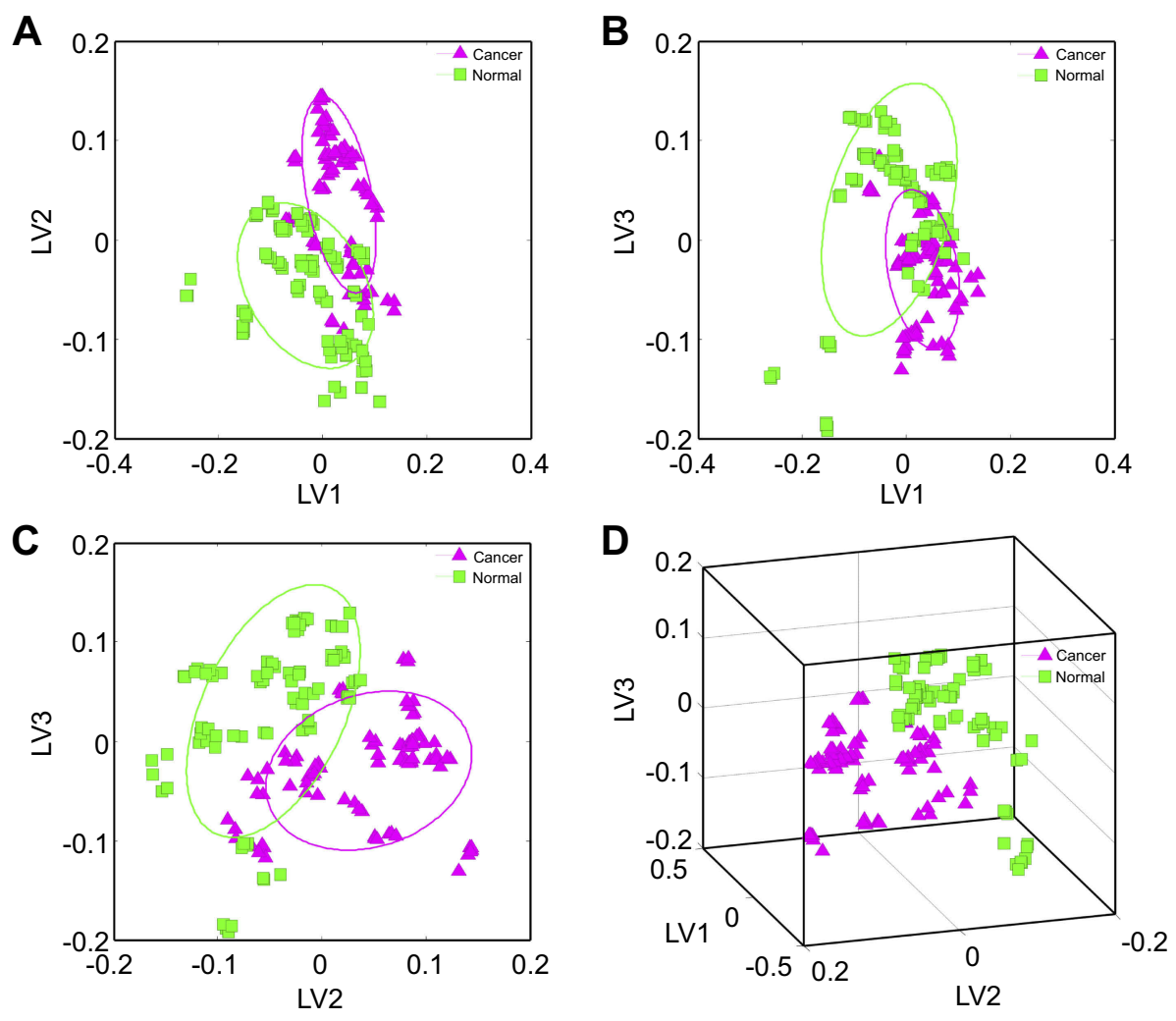

Figure 2 Discrimination of surgical aerosol mass-spectra data analyzed by PLS, demonstrating class separation between aerosol of cancerous tissue and adjacent normal tissue. Notes: (A) 2-D projection plots from PLS analysis of first and second latent variables (LVI and LV2); (B) 2-D projection plots from PLS analysis of LVI and LV3; (C) 2 D projection plots from PLS analysis of LV2 and LV3; (D) 3-D projection plots from PLS analysis of the first three latent variables (LVI-LV3) for the surgical aerosol Abbreviation: PLS, partial least squares.
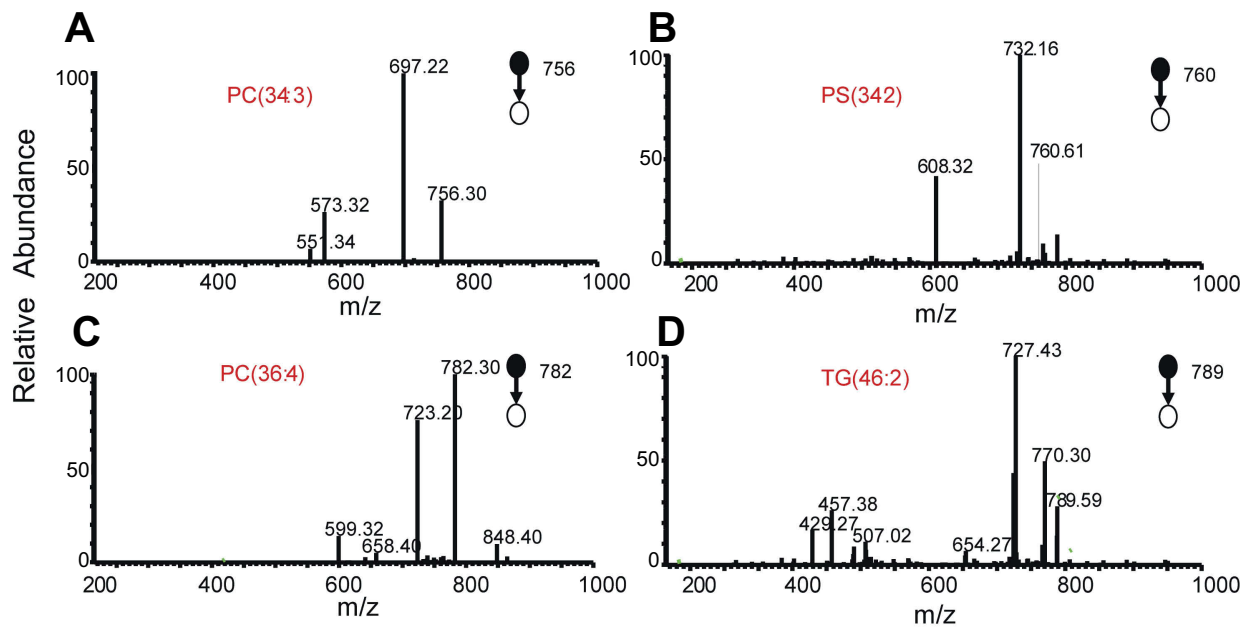

Figure 3 Mass spectra by collision-induced dissociation.

Notes: (A) PC (34:3; precursor ion $756.30 \mathrm{~m} / \mathrm{z}$ ); (B) PS (34:2; precursor ion $760.6 \mathrm{I} \mathrm{m/z);} \mathrm{(C)} \mathrm{PC} \mathrm{(36:4;} \mathrm{precursor} \mathrm{ion} 782.30 \mathrm{~m} / \mathrm{z})$; (D) TG (46:8; precursor ion 789.59 m/z). Abbreviations: PC, phosphatidylcholine; PS, phosphatidylserine; TG, triacylglycerols.

thus results are subjective. Therefore, there is an urgent need for in situ real-time identification of tissue features during surgery. Precision surgery for the management of lung cancer requires new technologies that can provide real-time, objective data on tumor margins for clinical decision-making. ${ }^{17}$ An ideal sample for discrimination of tumor margins during an operation is surgical aerosol, which is closely related to cell and molecular information. 
Chemical analysis by MS may be a perfect method to identification of tissue feature during an operation, as MS can catch trace changes in molecular information, which can be correlated with pathology. ${ }^{18-20}$ MS has been used for the investigation of tissue for more than four decades, providing numerical data for identification of tissue features. Recently, mass spectrometry analysis has development rapidly and a large number of ionization methods have been presented for discrimination of tissue feature. These methods including internal EESI-

$\mathrm{MS},{ }^{11}$ tip spray-ionization MS, ${ }^{4}$ matrix-assisted laser desorption-ionization $\mathrm{MS}^{21}$ desorption electrospray-ionization MS imaging ${ }^{22}$ and rapid evaporative-ionization MS. While it have been proved that these methods could identification tumor tissue from normal, there are some shortcoming of them (matrix-assistedlaser desorption-ionization MS and desorptionelectrospray-ionization MS require frozen tissue sections, internal EESI-MS and tipspray-ionization MS need sampling from patient). It has been reported that the rapid evaporative-ionization MS approach differentiates accurately between distinct histological and histopathological tissue types, with malignant tissue yielding chemical characteristics specific to histopathological subtypes, identifying lung tissue based on PA (34:1)-H, PE (34:2)- $\mathrm{NH}_{3}$, PE (36:1)- $\mathrm{NH}_{3}$, and PI (38:4)-H. ${ }^{6}$ In the current study, PS (34:2), PC (36:4), TG (46:2), and PC (34:3) were mutative in cancerous tissue aerosol from normal lung aerosol.

EESI-MS has demonstrated the ability to analyze liquids and gases. ${ }^{23,24}$ In the present study, the surgical aerosol was detected directly by EESI-MS. Our results demonstrated that EESI-MS enables direct chemical characterization of surgical aerosol from non-small-cell lung cancer. The results of PLS analysis revealed that a small fraction of compounds were different in surgical aerosol of cancer and normal tissue. On PLS analysis, a total of 208 surgical aerosol database entries from 26 patients were able reliably to distinguish between cancer tissue and adjacent normal tissue. Upon further analysis of statistically significant peaks, the results revealed that PS (34:2), PC (36:4), and TG (46:2) were increased, while PC (34:3) was decreased in cancer aerosol. At the molecular level, lipid metabolism is an important unit of tumor metabolism. ${ }^{22}$ Some tumors demonstrate a lipogenic phenotype, as membrane lipids are rapidly synthesized in tumor cells with high turnover. ${ }^{25}$ It has been reported that lipids are critical regulators of cancer, not only structurally but also in cell signaling between cancer and adjacent normal stroma. ${ }^{13}$ In our previous study, lipid metabolism in lung cancer confirmed that dipalmitoyl phosphatidylcholine and 1-palmityl-2-oleoyl phosphatidylcholine were decreased, but 2-oleoyl phosphatidylcholine and arachidonic acid-stearoyl phosphatidylcholine increased in lung squamous-cell carcinoma tissue relative to normal tissue. ${ }^{4}$ In the present study, lipids were altered in surgical aerosol from lung cancer tissue. The relative abundance of PS (34:2), PC (36:4), TG (46:2) was increased, while PC (34:3) was decreased in cancer aerosol. These lipids may be consideraed biomarkers in surgical aerosol of non-small-cell lung cancer.

This is the first study to apply EESI-MS to discriminate lung cancer during surgery. The aim of the study was to validate the diagnostic potential of the surgical aerosol. With EESI-MS, trace changes in lipids werecaught. After PLS analysis, MS data of surgical aerosol distinguish cancerous tissue from adjacent normal tissue. EESI-MS coupled with PLS analysis selected the most significant ion peaks for discriminating between cancer and adjacent normal tissue. Different metabolite IDs for surgical aerosol were identified following a search of the Human Metabolome Database. Result revealed that surgical aerosol might contain biomarkers for identification of lung cancer and adjacent normal tissue. In this analysis, it hasbeen proved that surgical aerosol is able to identify cancer during an operation. The authors know the limitations of this study. The sample was small, the mechanisms of discrimination not yet well understood, and we collected only ex vivo experimental data. Although this study did not achieve real-time and in situ differentiation between cancer and normal tissue in surgery, the diagnostic potential of surgical aerosol was clarified.

\section{Conclusion}

With coupling PLS and EESI-MS analysis of surgical aerosol, MS data for non-small-cell lung cancer was able to be distinguished clearly from normal tissue. The surgical aerosol might contain biomarkers for identification of cancer and normal tissue. The altered lipid profile in cancerous aerosol is significant for further study. Detecting surgical aerosols may be a viable method for discrimination of tumor margins during operations.

\section{Abbreviation list}

PLS, partial least squares; EESI, extractive electrospray ionization; MS, mass spectrometry; PC, phosphatidylcholine; PS, phosphatidylserine; TG, triacylglycerol. 


\section{Ethics approval and informed consent}

This study was approved by the Medical Ethics Committee of the hospital's institutional review board. All clinical investigations were conducted according to the principles expressed in the Declaration of Helsinki.

\section{Acknowledgments}

We thank Dr Wang Yiming (Department of Cardiothoracic Surgery, Second Affiliated Hospital of Nanchang University) for providing patient data. We also thank Dr Wu Liqing (Department of Pathology, Second Affiliated Hospital of Nanchang University) for providing pathological data. We also thank the patients. This work was supported by grants from the National Natural Science Foundation of China (81560345 and 81860379), Preeminence Youth Fund of Jiangxi Province (20162BCB23058), China Postdoctoral Science Foundation Grant (2017M610401), Science and Technology Planning Project at the Department of Science and Technology of Jiangxi Province, China (20151BBG70165 and 20171BAB205075).

\section{Disclosure}

The authors report no conflicts of interest in this work.

\section{References}

1. Siegel RL, Miller KD, Jemal A. Cancer statistics, 2018. CA Cancer J Clin. 2018;68(1):7.

2. Chen W, Zheng R, Baade PD, et al. Cancer statistics in China, 2015. CA Cancer J Clin. 2016;66(2):115. doi:10.3322/caac.21338

3. Miller, KD, Siegel RL, Lin CC, et al. Cancer treatment and survivorship statistics, 2016. CA Cancer J Clin. 2016;66(4):271. doi:10.3322/ caac. 21349

4. Wei Y, Chen L, Zhou W, et al. Tissue spray ionization mass spectrometry for rapid recognition of human lung squamous cell carcinoma. Sci Rep. 2015;5:10077. doi:10.1038/srep10077

5. D‘Addario G, Früh M, Reck M, et al. Metastatic non-small-cell lung cancer: ESMO clinical practice guidelines for diagnosis, treatment and follow-up. Ann Oncol. 2016;21 Suppl 5(suppl_5):v116-v119.

6. Balog J, Sasi-Szabó L, Kinross J, et al. Intraoperative tissue identification using rapid evaporative ionization mass spectrometry. Sci Transl Med. 2013;5(194):153-154. doi:10.1126/scitranslmed.3005623

7. S S C, Nakano T, Okamoto T. Usefulness of intraoperative computer tomography-assisted thoracoscopic segmentectomy for small-sized lung cancer. Atcs. 2015;22(4):261.
8. John ERS, Alkhudairi R, Ashrafian H, et al. Diagnostic Accuracy of Intraoperative Techniques for Margin Assessment in Breast Cancer Surgery: a meta-analysis. Ann Surg. 2016;265(2):300. doi:10.1097/ SLA.0000000000001897

9. John ER, Balog J, Mckenzie JS, et al. Rapid evaporative ionisation mass spectrometry of electrosurgical vapours for the identification of breast pathology: towards an intelligent knife for breast cancer surgery. Breast Cancer Res. 2017;19(1):59. doi:10.1186/s13058-017-0845-2

10. Gong JW. Application of Mass Spectrometry in Biology and Physiology [PhD thesis]. Cincinnati, OH: University of Cincinnati; 2016.

11. Zhang H, Gu H, Yan F, et al. Direct characterization of bulk samples by internal extractive electrospray ionization mass spectrometry. Sci Rep. 2013;3(6148):2495. doi:10.1038/srep02495

12. Ina A, David M. Advantages and pitfalls of mass spectrometry based metabolome profiling in systems biology. Int J Mol Sci. 2016;17(5):E632

13. Balog J, Kumar S, Alexander J, et al. In vivo endoscopic tissue identification by rapid evaporative ionization mass spectrometry (REIMS). Angew Chem Int Ed. 2015;54(38):11059-11062. doi:10.1002/anie.201502770

14. Chen H, Wortmann A, Zenobi R. Neutral desorption sampling coupled to extractive electrospray ionization mass spectrometry for rapid differentiation of biosamples by metabolomic fingerprinting. J Mass Spectrom. 2007;42(9):1123-1135. doi:10.1002/jms.1282

15. Hirsch FR, Scagliotti GV, Mulshine JL, et al. Lung cancer: current therapies and new targeted treatments. Lancet. 2016;389(10066):299. doi:10.1016/S0140-6736(16)30958-8

16. Vimal M. A study on accuracy of frozen section diagnosis and turnaround time. Int J Health Sci Res. 2015;5(12):138-142.

17. Mirnezami R, Rcs M, Nicholson J, et al. Preparing for precision medicine. $N$ Engl J Med. 2012;366(6):489-491. doi:10.1056/ NEJMp1114866

18. Wang H, Manicke NE, Yang Q, et al. Direct analysis of biological tissue by paper spray mass spectrometry. Anal Chem. 2011;83 (4):1197-1201. doi:10.1021/ac103150a

19. Liu J, Cooks RG, Ouyang Z. Biological tissue diagnostics using needle biopsy and spray ionization mass spectrometry. Anal Chem. 2011;83(24):9221-9225. doi:10.1021/ac202626f

20. Takats Z. Analysis of dried blood spot samples by high resolution mass spectrometry - from newborn screening to cancer diagnostics. Clin Biochem. 2014;47(9):699. doi:10.1016/j.clinbiochem.2014.05.014

21. Gao W, Ou G, Feng X, Liu B-F, Zhang H, Liu X. Matrix-assisted laser desorption/ionization mass spectrometry analysis of glycans with co-derivatization of asparaginyl-oligosaccharides. Anal Chim Acta. 2015;896:102. doi:10.1016/j.aca.2015.09.028

22. Black DM. Application of desorption electrospray ionization mass spectrometry imaging in breast cancer margin analysis. Proc Natl Acad Sci US A. 2014;111(42):15184-15189. doi:10.1073/pnas.1408129111

23. Chen H, Yang S, Li M, et al. Sensitive detection of native proteins using extractive electrospray ionization mass spectrometry. Angew Chem. 2010;49(17):3053. doi:10.1002/anie.201002580

24. Pan S, Tian Y, Li M, et al. Quantitative detection of nitric oxide in exhaled human breath by extractive electrospray ionization mass spectrometry. Sci Rep. 2015;5:8725. doi:10.1038/srep08725

25. Santos CR, Schulze A. Lipid metabolism in cancer. FEBS J. 2013;1831(10):1497-1498.
Cancer Management and Research

\section{Publish your work in this journal}

Cancer Management and Research is an international, peer-reviewed open access journal focusing on cancer research and the optimal use of preventative and integrated treatment interventions to achieve improved outcomes, enhanced survival and quality of life for the cancer patient.
The manuscript management system is completely online and includes a very quick and fair peer-review system, which is all easy to use. Visit http://www.dovepress.com/testimonials.php to read real quotes from published authors. 